Under the allocation system, the nation's ro largest universities scooped up two-thirds of the chairs, while the biomedical and natural sciences each received $40 \%$ of the positions and the social sciences and humanities just $20 \%$. In the interest of promoting more differentiation and specialization by universities, each was also required to situate their chairs within the context of an institutional strategic plan which sketched the disciplines in which it would specialize.

Over the course of the program's lifetime, various criticisms have been leveled to the effect that it was biased against women, that it encouraged faculty poaching by larger universities, and that it created elites within the academy, as well as "have" and "have not" disciplines.

Gaffield argues that such concerns have since been dispelled. "My sense is that the constant poaching that some people said would ensue has not happened," he says. "Sure there is some movement but it has not become a problem and we have heard very little criticism of that now."

Program administrators say that as of November $2007, \mathrm{I} 4.4 \%$ of all chairs were awarded to ex-patriots; $12 \%$ were awarded to recipients raided from other Canadian universities and $7 \%$ to recipients coming from outside a university. Nearly $22 \%$ of recipients came from outside the country and $58.5 \%$ of chairs were awarded to existing faculty within a university, while $22 \%$ of chairholders are women.

Gaffield also says there's absolutely no doubt that the program has resulted in significant specialization within the nation's universities.

"What we've all found on campus is that we want to have a strong foundation across our fields but clearly if we're going to make significant contributions to research, you really have to focus. You can't be internationally outstanding in everything. Even the biggest universities, and this is true whether it is Oxford or Harvard or the University of Toronto or any university, you're just not going to be internationally outstanding in everything." Wayne Kondro, CMAJ

DOI:I0.1503/cmaj.08054I

\section{Inducing seizures}

\section{among seniors}

1 pression, psychotherapy and medications offer little respite, which prompts many psychiatrists to turn to electroconvulsive therapy, particularly to relieve psychosis or thoughts of suicide.

It's been anecdotally suggested by several Canadian physicians that inducing seizures in the brains of Canadian seniors is rapidly becoming the norm in the treatment of the elderly for depression. Quantifying that trend, though, is somewhat problematic because of provincial differences in the reporting of data to the Canada Institute for Health Information (CIHI). There is some evidence to suggest, however, that electroconvulsive therapy is, in fact, now more often administered on an outpatient basis and more often to seniors.

The popularity of electroconvulsive therapy has ebbed and flowed over the course of its 70-year history, but the treatment is now well accepted in Canada, says Barry Martin, head of the electroconvulsive therapy service at the Center for Addiction and Mental Health in Toronto, Ontario.

Data on its current use are incomplete, although CIHI conservatively estior some patients with major de- mates that annual administration of electroconvulsive therapy has been relatively unchanged, at about 5000 procedures per year, since 2002. But CIHI lacks information about outpatient procedures performed in Quebec and Alberta, and data for hospitals outside the Winnipeg area prior to the 2004/05 fiscal year.

The treatment's usage also appears to have oscillated during the last 25 years. In Quebec, the number of patients who received electroconvulsive therapy increased from 455 to 87 I between I983 and 2003; 68I patients were treated last year. During the late ig8os and early I99os, its use on an inpatient or day surgery basis in Manitoba hospitals declined, but peaked at 482 patients in I999. In Ontario, treatments have also been on the rise, from 7800 to ro 800 between I999 and 2005 .

According to Canada-wide statistics, electroconvulsive therapy is increasingly being delivered on an outpatient basis, eliminating the need for overnight stays in unfamiliar institutions and reducing the risk of exposure to hospital infections.

"If the patient is well enough not to require inpatient hospitalization, it is better to have it done [as an outpatient] and go home," says Martin. Inpatient electroconvulsive therapy accounted for half the treatments provided in Canada in 2002 , but only $36 \%$ in 2005 (Figure I). The nationwide stats also

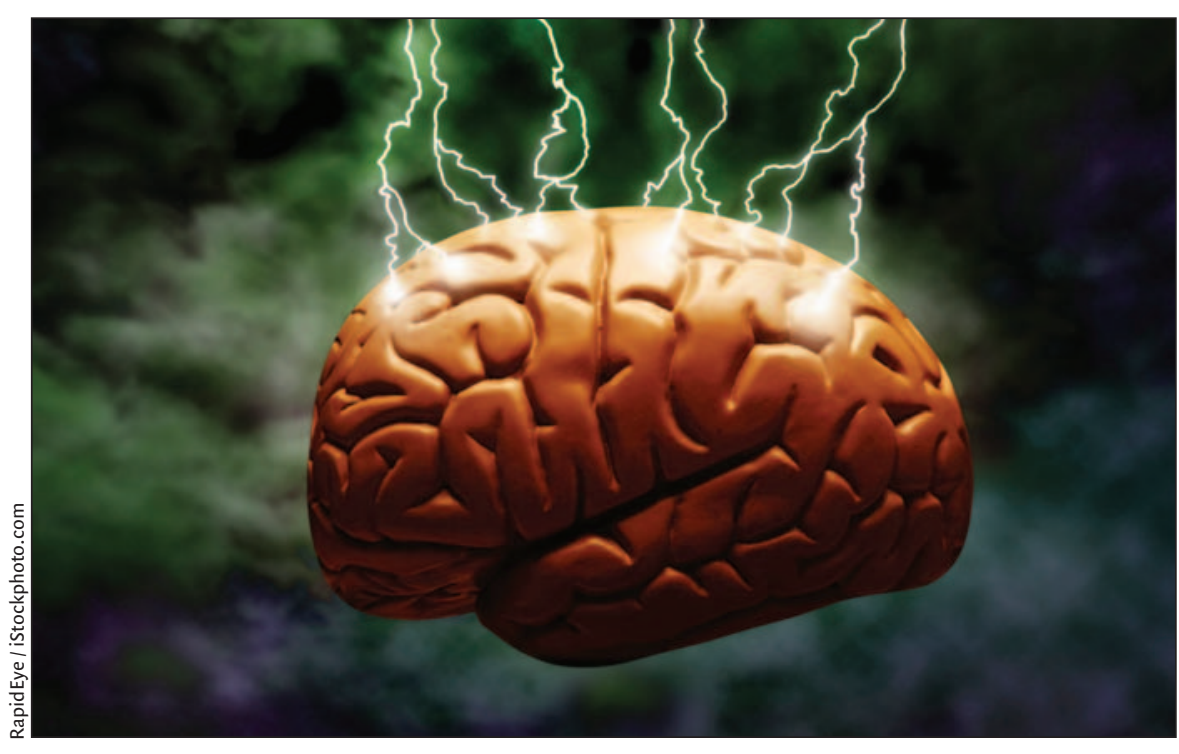

Electroconvulsive therapy is increasingly being delivered on an outpatient basis and being administered to seniors as treatment for depression. 
confirm that electroconvulsive therapy is being given to seniors more often. Patients over the age of 70 received $30 \%$ of all electroconvulsive therapy procedures in 2005, as compared with $23 \%$ in 2002 (Figure 2). Caroline Gosselin, a geriatric psychiatrist and clinical professor in University of British Columbia Department of Psychiatry says that's because depression is common among the elderly and is more likely to lead to suicide; men over the age of 80 have the highest suicide rate among Canadians.

"Sometimes an older person won't respond to an antidepressant. The depression becomes worse and then it becomes life threatening. ECT [electroconvulsive therapy] works quickly," Gosselin says.

Some advocates are concerned that it is being overused in the elderly. "Certainly there is a lot of depression [in the elderly], but my opinion is that ECT [electroconvulsive therapy] is an easier method than trying medications and it adds a measure of control," Juli Lawrence says. Lawrence, who had electroconvulsive therapy, established ECT.org, a website that provides information about the treatment.

Patients offer mixed responses about electroconvulsive therapy. Some claim to have been well informed about the treatment and the risks involved, and that it succeeded in relieving their depression. Others say they were forced into it, were not fully informed of the potential memory loss or allowed to stop treatment at their will.

Murray Enns, a professor and head of psychiatry at the University of Manitoba, says practices are now differerent. "Even textbooks written in the I950s reflected the attitude that the doctor ought not to worry the patient about side effects." The most common complaint associated with electroconvulsive therapy is memory impairment. Patients often wake up confused immediately after the treatment. They may have trouble remembering some past events and some have difficulty forming new memories for a short time. When unilateral electroconvulsive therapy is used, the memory effects are generally less severe.

"It is a risk-benefit analysis. Gener-

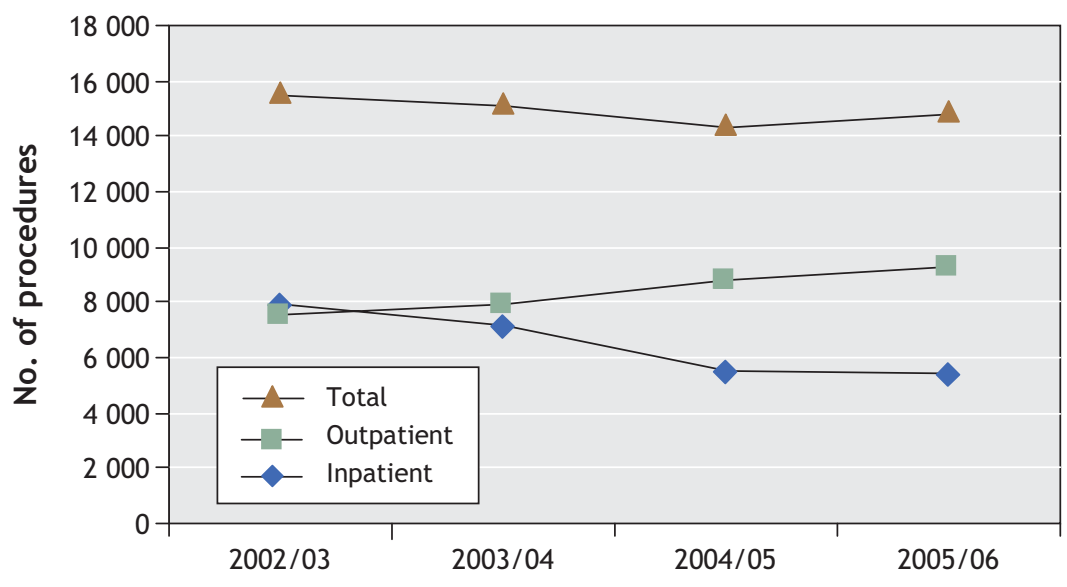

Figure 1: Total number of electroconvulsive therapy procedures administered in Canada, by inpatient and outpatient basis.

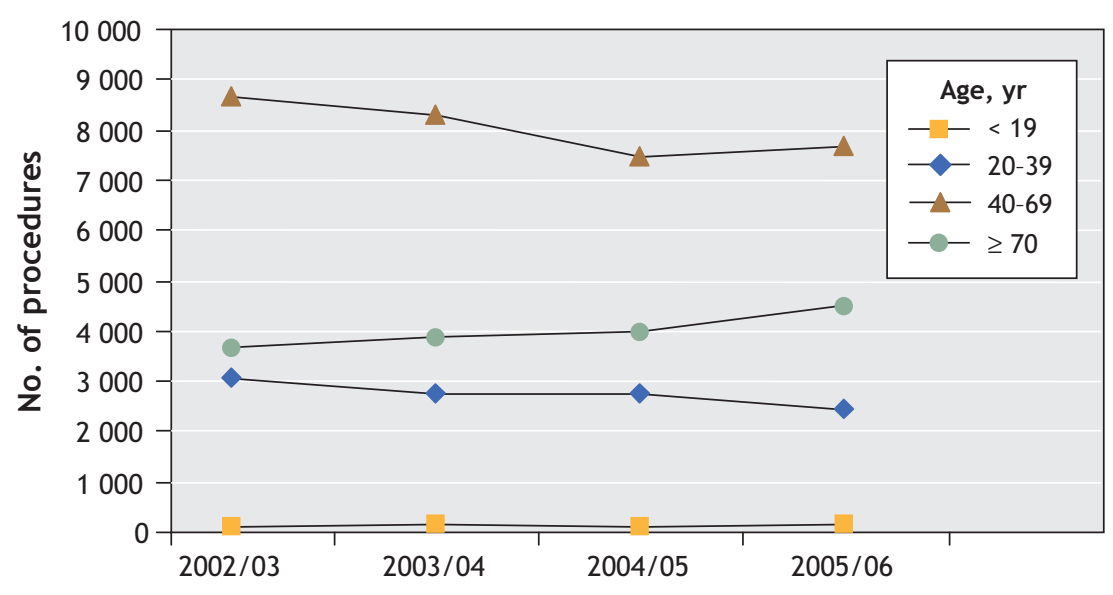

Figure 2: Frequency of electroconvulsive therapy procedures by age group.

ally people accept it because it is much less debilitating than the ongoing depression," says Martin. "We make it very clear when obtaining consent that there is a risk of patchy loss of memory. There is a full recovery of anterograde memory - the ability to form new memories. If there wasn't, this procedure would never have lasted."

Sue Clark says she received 5 electroconvulsive therapy treatments in I973 when she was I7 years old, but didn't benefit from them. "I suffer from permanent memory loss and have trouble learning new things," she says. She's concerned that patients aren't being fully informed of the potential side effects of electroconvulsive therapy, including the risk of memory loss.
Some allege that electroconvulsive therapy causes brain damage. The proof, they say, is in the long term effects it has on memory. But there is no evidence to support that, says Nick Delva, head of the department of psychiatry at Dalhousie University. "When there is brain damage, as there is with a stroke, there are chemicals that can be found in the blood. They've been measured [following electroconvulsive therapy] and they're not there."

But a 2007 study confirmed that electroconvulsive therapy can have some long-term memory effects. Harold Sackeim, chief of the Department of Biological Psychiatry at the New York State Psychiatric Institute and professor at the College of Physicians 
and Surgeons at Columbia University, found that some older forms of electroconvulsive therapy were still in use in the New York metropolitan area. "The use of sine wave stimulation and the [bilateral] electrode placement were both associated with greater short- and long-term deficits," he and his colleagues write in Neuropsychopharmacology. "There appears to be little justification for the continued first-line use of BL ECT [bilateral electroconvulsive therapy] in the treatment of major depression."

Delva is conducting a national electroconvulsive therapy survey called CANECTS, which aims to ultimately reveal how many facilities now offer electroconvulsive therapy, which conditions are being treated, what type of equipment is being used, what consent processes are being followed and what information is being revealed to pa- tients. Teaching, budgets and patient access will also be assessed.

"There was no information of this sort for Canada," he says. Preliminary results indicate electroconvulsive therapy is now offered at 177 sites.

International data comparing the use of electroconvulsive therapy as a treatment for depression among seniors are unavailable. But what limited international data exist on overall electroconvulsive therapy use suggest that rates are lower in Canada than elsewhere $U$ Affect Disord 2006;90[I]:67-7I). The overall rate in Denmark was 30.5 per 10000 in 1999, II.4 in India in 2002, 10.9 in Great Britain in 1999, 6.8 in Belgium in 2003 and I.8 in the Netherlands in I999. Using CIHR and Statistics Canada data, the rate for Canada was 4.6 in 2005. Hannah Hoag, Montréal, Que.

DOI:I0.I503/cmaj.080360

\section{Notes on electroconvulsive therapy}

Although electroconvulsive therapy is accepted by the Canadian and American psychiatric associations as a treatment for major depression and bipolar disorder, it has a checkered history. It once was administered without muscle relaxants or anaesthetics. Full body convulsions caused serious complications, including changes in heart rhythm and vertebral compression fractures. Early generations of electroconvulsive therapy machines delivered electricity at much higher doses, and in a waveform later found to be less efficient at inducing brain seizures and more likely to cause memory impairment. In the I950s to I970s, during which consent procedures were less sophisticated, there was misuse; for example, to "treat homosexuality" (BMJ 2004;328:955-6). Many activists continue to demand that it be banned.

Electroconvulsive therapy delivery has been significantly refined, while the risk of fracture has been virtually eliminated. Patients receive anaesthesia, a muscle relaxant, ventilation and oxygen. Two electrodes are placed on the scalp, either on both temples (bilateral electroconvulsive therapy) or on one side of the head (unilateral electroconvulsive therapy). A pulse of electricity is typically used for a few seconds.

Some people respond rapidly, requiring only 5 or 6 treatments, Enns says. Patients usually take an antidepressant and a mood stabilizer to delay a relapse; a 2006 study found that electroconvulsive therapy — I dose every 4 to 6 weeks can have similar effects (Arch Gen Psychiatry 2006:63 [12]:1337-44).

Some studies in animal models suggest that electroconvulsive therapy may in fact produce new brain cells. It increases the expression of brain-derived neurotrophic factor, a peptide that has been shown to support neuron growth in brain regions key to the regulation of mood and behaviour (The Pharmacogenomics Journal 2008;8:I0I-I2). Decreased serum brain-derived neurotrophic factor levels have been reported in depressed patients (Psychiatry Research 2002;109[2]:143-8). Other studies have found that plasma BDNF levels of patients receiving electroconvulsive therapy for major depression increased following treatment (UClin Psychiatry 2007;68[4]:512-7).

\section{The strains of Ebola}

I nside tiny Kikyo Health Centre nestled high in the Rwenzori Mountains of Uganda, a sheet on the office wall entitled "Weekly epidemiological cases," tracks incoming patients. The list gives testimony to the challenges faced when a ro-bed facility with no doctor on staff has to cope with medical conditions that, in Western countries, rarely surface anywhere but in text books: acute flaccid paralysis, rabies, dysentery, Guinea worm, meningitis.

And now, added to the bottom of the list, in big black block letters, is "EBOLA."

This health centre, reached only by foot across the Bwamba Pass or by an arduous mountain drive from Bundibugyo town, was the epicenter of an Ebola outbreak late in 2007 that killed 37 and confounded medical experts for months, until they realized what they had on their hands was a new strain of the deadly virus.

While the experts grappled with the "mysterious illness," people in western Uganda began dying of it in August 2007. It was not until Nov. 29, 2007, that the government publicly announced the Ebola outbreak. In all, 9I of the 149 reported Ebola cases that surfaced in western Uganda between August 2007 and January 2008 were admitted to Kikyo, where staff struggled to manage the outbreak without even rubber gloves or face masks.

"We were not so much prepared for an epidemic of that proportion," says Julius Monday, an animated man who runs the health centre and is now something of a local legend for having

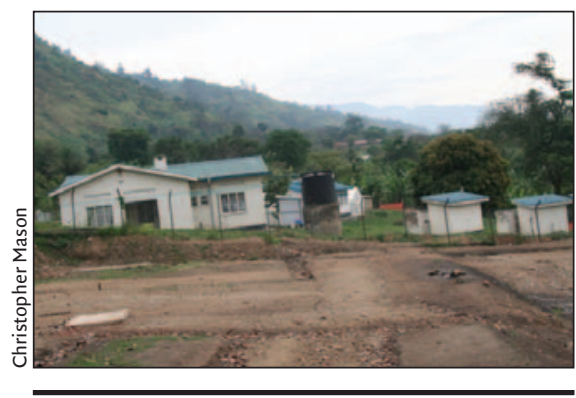

Kikyo Health Centre, high in the Rwenzori Mountains overlooking the Democratic Republic of Congo. 